\title{
VOLUNTARY ACTIVITIES AND ONLINE EDUCATION FOR DIGITAL HERITAGE INVENTORY DEVELOPMENT AFTER THE GREAT EAST JAPAN EARTHQUAKE
}

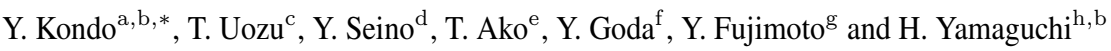 \\ ${ }^{a}$ Dept. of Computer Science, Tokyo Institute of Technology, Tokyo 152-8552 Japan \\ kondo@archaeo.cs.titech.ac.jp \\ b Japan Society for the Promotion of Science \\ ${ }^{\mathrm{c}}$ Research Institute of History, Otemae University, Nishinomiya 662-0965 Japan \\ tomouozu@otemae.ac.jp \\ ${ }^{d}$ Dept. of Cultural Coexistence, Kyoto University, Kyoto 606-8501 Japan \\ seinoyoichi@gmail.com \\ ${ }^{\text {e }}$ Museum, Tokyo Institute of Technology, Tokyo 152-8550 Japan \\ ako.t.aa@m.titech.ac.jp \\ ${ }^{\mathrm{f}}$ Research Center for Higher Education, Kumamoto University, Kumamoto 860-8555 Japan \\ ygoda@kumamoto-u.ac.jp \\ ${ }^{g}$ Dept. of Geography, Nara University, Nara 631-8502 Japan \\ yfujimot@ daibutsu.nara-u.ac.jp \\ h Tezukayama University, Nara 631-8501 Japan \\ yamahirog@gmail.com
}

KEY WORDS: Great East Japan Earthquake, disaster-damaged cultural heritage, voluntary initiative, digital restoration of library catalogue, e-Learning, disaster-related heritage studies

\begin{abstract}
:
Consortium for Earthquake-Damaged Cultural Heritage (CEDACH) is a voluntary initiative launched just after the Great East Japan Earthquake on 11 March 2011. The consortium is developing a social network between local cultural resource managers restoring disaster-damaged cultural heritage on one side and remote researchers including historians, archaeologists and specialists of cultural information studies on the other side, in order to facilitate collaborative projects. This paper presents three projects in which CEDACH contributed to the development of a digital inventory for disaster-damaged heritage management through web-based collaborations by self-motivated workers. The first project, CEDACH GIS, developed an online archaeological site inventory for the disaster area. Although a number of individuals voluntarily participated in the project at the beginning, it gradually stagnated due to limited need for local rescue archaeology. However, the experience of online-based collaborations worked well for the second project proposed by local specialists, in which CEDACH restored the book catalogue of a tsunami-devastated research library. This experience highlighted the need for online education to improve information and communication technologies (ICT) skills of data builders. Therefore, in the third project called CEDACHeLi, an e-Learning management system was developed to facilitate learning the fundamental knowledge and techniques required for information processing in rescue operations of disaster-damaged cultural heritage. This system will contribute to improved skills and motivation of potential workers for further developments in digital heritage inventory.
\end{abstract}

\section{INTRODUCTION}

The Great East Japan Earthquake on 11 March 2011 caused tremendous damage to various historical documents and cultural heritage. Currently, the restoration projects are still only halfway implemented. Among these projects, the Consortium for the Earthquake-Damaged Cultural Heritage $(\mathrm{CEDACH})$ is developing a social network that bridges the following three groups: The first group consists of local cultural resource management (CRM) officers, researchers, NPOs (non-profit organisations), and citizens who are directly engaged in the restoration of damaged and endangered cultural properties. The second group involves researchers and CRM officers who were sent from other regions to the disaster area for extending help. The third comprises remote researchers, including historians, archaeologists, and specialists of cultural information studies as well as other members of the public willing to provide support with their professional skills and/or manpower despite their difficulty to stay in the disaster-hit area to provide direct support. CEDACH decided to make use of web-based services to develop an open and low-cost social infrastructure that facilitated the collaboration among these three groups, with as many advocates as possible joining the projects without concerns about their own business and geographical re- strictions (Fujimoto et al., 2011, Kondo et al., 2012, Okamura et al., 2013). In the process of recognising local needs, CEDACH anticipated that a digital heritage inventory (Myers et al., 2012) should be implemented for handling a large-scale and unorganised database of disaster-damaged cultural heritage. This would be accomplished with the help of advanced information and communication technologies (ICT) and manpower derived from selfmotivated volunteers. This paper reports on three projects in which CEDACH contributed to developing a digital inventory for disaster-damaged heritage management, based on the abovementioned needs-(1) CEDACH GIS, an attempt to develop a web-based heritage site database system for the disaster area; (2) digital restoration of the book catalogue from a tsunami-devastated research library; and (3) CEDACHeLi, an e-Learning curriculum for information processing in rescue projects of disaster-damaged cultural heritage.

\section{CEDACH GIS}

Just after the launch of this initiative, CEDACH started developing a geospatial information system called CEDACH GIS. This system was implemented to facilitate rescue operations, conservation, and restoration of disaster-damaged cultural heritage by 


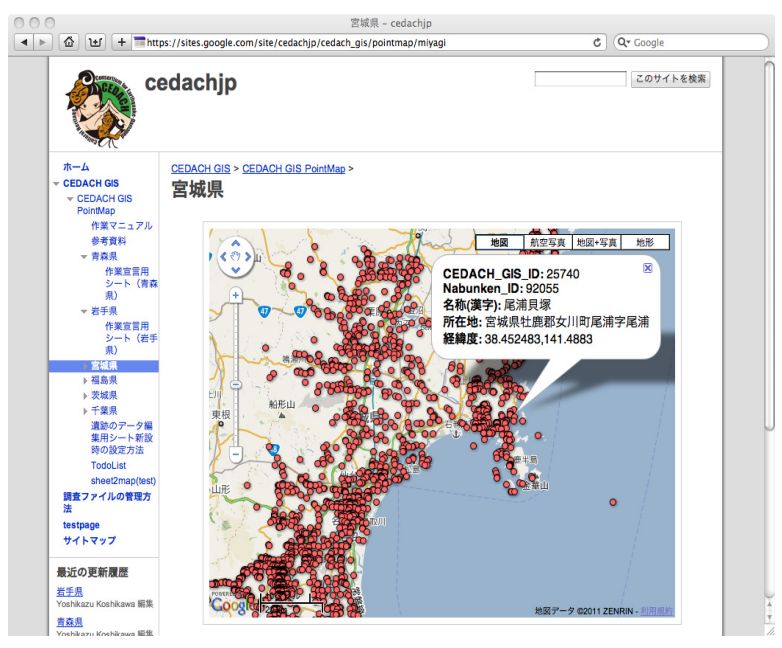

Figure 1: The interface of the CEDACH GIS

integrating various sorts of information into a unified database system with geospatial information in order to understand where, what, and how the cultural properties were present. For this purpose, the system handled a large-scale dataset, including digital elevation models, airborne and satellite images captured before and after the tsunami, and the locations and attributes of archaeological sites and monuments (Fujimoto et al., 2011, Kondo et al., 2012). Since the data collection and validation required a large amount of collaborative manpower, the CEDACH GIS employed a web-based working platform and open data source so that selfmotivated people could easily participate in the work.

At the beginning of data accumulation, $\mathrm{CEDACH}$ listed archaeological sites and monuments in the disaster areas. Approximately 50 self-motivated workers, including a number of geography students, participated in this project to check the geocoordinates of sites registered in the nationwide database hosted by the Nara National Institute for Cultural Properties. This work employed SaaS (Software as a Service) available online. Sites were mapped by means of Google Fusion Tables and Google Maps (Figure 1), and the locations were then cross-checked with reference to the readymade online digital maps provided by the Geospatial Information Authority of Japan.

The CEDACH GIS project faced difficulties when it looked into the needs of practical applications of the local CRM projects in the disaster area. In the early stages, the restoration processes for damaged cultural properties were not yet determined. Therefore, CEDACH started developing the system, anticipating that GIS would be required for restoration projects. However, it turned out later that restoration proceeded more quickly than expected. Since most of the archaeological heritage sites were buried underground, they were minimally damaged. In contrast, a number of historical monuments, architectural remains, museums, and public storage facilities for local historical documents, folklore materials, and archaeological objects in the coastal areas were devastated by the tsunami. These were not always registered in the newly-implemented archaeological site database. In theory, there might have been some need for an archaeological database in rescue archaeology before construction work over the course of restoring social infrastructure. However, local CRM officers did not actually rely on the new mapping system. When they were asked to conduct an archaeological survey before constructions, as stipulated by the Law for the Protection of Cultural Properties, they tended conducted it as quickly as possible, with their customary conventional manner. The local governments also started applying the all-in-one GIS, including urban planning and risk management. The need for CEDACH GIS then decreased moderately, and this led to the unfeasibility damaging the workers'

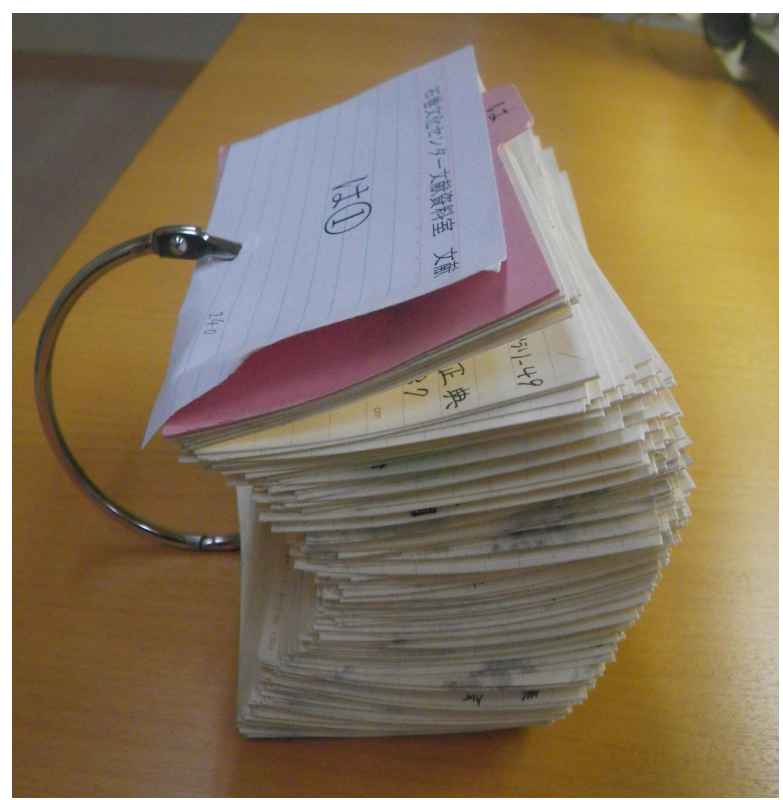

Figure 2: A set of book catalogue cards

motivation. This vicious cycle finally resulted in stagnation of the development of CEDACH GIS. At that time, CEDACH received the offer to restore a book catalogue, as reported below.

\section{DIGITAL RESTORATION OF THE LIBRARY CATALOGUE}

The storage of Ishinomaki Culture Center, a municipal museum at Ishinomaki, Miyagi prefecture, was devastated and heavily contaminated by the tsunami and resulting debris (Kaner et al., 2011, Uozu and Seino, 2013). There was previously a research library in the Curatorial Division, which was known by local researchers (who typically worked in the public sector) as an outstanding book collection of history, art history, archaeology, and folklore of the region. The collection previously contained a number of municipal history series, exhibition catalogues, art books, excavation reports, and ethnographic reports. The book inventory was managed with paper-based cards. After the tsunami, items stored in the museum were primarily rescued and cleaned by the Bunkazai Rescue Project, a governmental task force (Okamura et al., 2013). The top priority of their mission was to rescue the registered items, but the books were excluded because they were not regarded as part of the official museum collection. Later, rescue technicians from the Miyagi Shiryounet (the NPO Miyagi Network for Preserving Historical Materials) dealt with them. However, they had to discard most of the waterlogged and contaminated books because it was impossible to restore them. The exception included 1,500 undamaged books. The card shelf was also waterlogged, although they nevertheless found approximately 19,000 pieces of book catalogue cards left mostly intact (Figure 2). It turned out that the cards were the only source for restoring the original inventory when municipalities, museums, universities, and other institutions offered to donate the same books. The restoration was much desired, but it was beyond the capacity of the Miyagi Shiryounet in terms of technology, manpower, and experience. Therefore, they suggested that CEDACH accomplish the project through collaboration with some local researchers who were involved with both initiatives. The CEDACH agreed to accomplish the project and received the cards at its main workspace in the Research Institute of History, Otemae University, Hyogo prefecture in western Japan.

CEDACH organised a project team with self-motivated volunteers to digitise and archive the book catalogue cards (CEDACH 


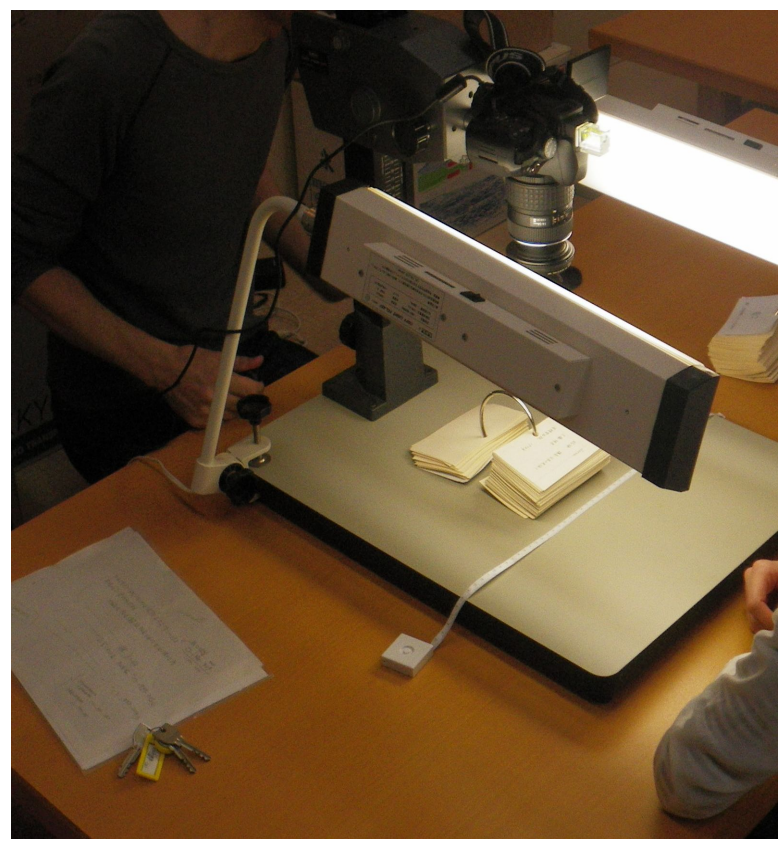

Figure 3: Book catalogue cards being digitally photographed; volunteers were worked in pairs

Jimukyoku, 2012, Uozu and Seino, 2013). The SaaS was employed for this project because the preceding CEDACH GIS project proved that its availability in the remote working environments was essential for group work based on the social network. The workflow began with photographing the cards with a digital camera (Figure 3). The photographs were processed by workers in pairs, based on the policy that each worker should experience as many parts of the workflow as possible. The pictures were then uploaded to an online photo album service. The next step was to input bibliographic information to a table, based on reading individual cards in the photographs. Web-based spreadsheets in Google Drive were used for this work. Both photo albums and spreadsheets were managed by URI (Uniform Resource Identifier) to indicate the permanent location of data on the Internet. URI made it possible for individual members to work remotely, such as at home or school. When new workers joined the project, the project manager pointed out the URIs in a spreadsheet in the unified format along with pictures of catalogue cards. Workers carried out their individual tasks, sometimes referring to online manuals. During data entry, errors occasionally occurred because some images were too coarse to recognise letters. In order to reduce errors, the data entries were cross-checked by other workers in possession of the actual cards at Otemae University. After the edit was completed, spreadsheets were integrated into one main sheet that underwent a final check and retouch of the format.

The project team started calling for volunteers on 17 April 2012, and completed the primary data input on 24 January 2013. In total, 52 self-motivated individuals participated in the project. The work was successfully achieved by remote collaborations where security was easily maintained, since there was still some risk of information leaks when malicious hackers knew the URIs. In the process of cross-checking, duplicate records were removed. As it turned out in the end, the library previously stored approximately 15,000 books, which meant that only $10 \%$ of the collection survived. The cross-checking will finish in summer of 2013. The catalogue cards and spreadsheets will be returned to the Ishinomaki Culture Center at the end of the project.

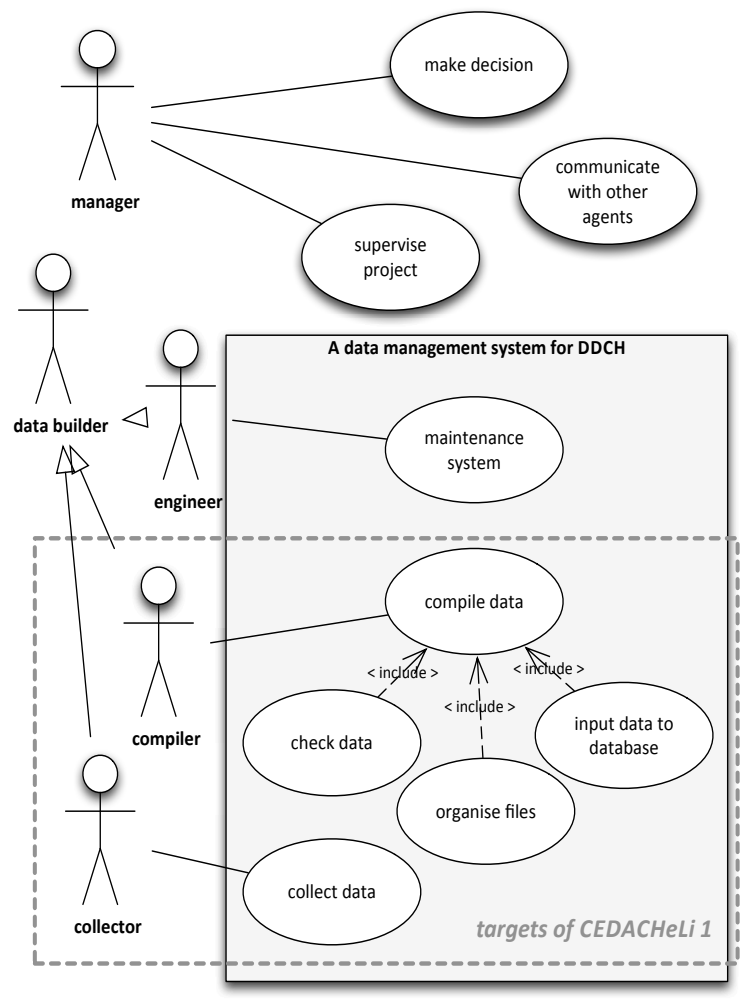

Figure 4: Flowchart illustrating how the CEDACHeLi is used

\section{E-LEARNING}

The experience of the GIS and book catalogue projects showed the need for a certain level of ICT skills for those who participated in the digital heritage inventory development. Such skills are usually acquired through on-the-job training, but it was thought that self-learning could also be effective in developing workers' knowledge and skills. Based on this conclusion, CEDACH started developing an e-Learning system, or an online self-learning environment in July 2012 to provide an opportunity to acquire knowledge, technical skills, and practical tips on information processing during the rescue operations of disaster-damaged cultural heritage. It was also expected that an Internet-based system could facilitate individual learning remotely, which was suitable for the online social network of CEDACH.

The e-Learning was named CEDACHeLi, an abbreviation of CEDA$\mathrm{CH}$ e-Learning for information processing. Trainees, or potential users of the CEDACHeLi, may have various motivations and different levels of ICT skills. The first curriculum (hereafter called CEDACHeLi 1) targeted the largest groups of workers who handled data, i.e. the primary data collectors and secondary data compilers who input data to the database (Figure 4). It was assumed that the majority of workers in these cases had limited ICT knowledge and skills. The curriculum was designed to provide an introduction to ICT-based support for the rescue operations.

In theory, there could be two other groups involved with the ICTbased heritage rescue projects (Figure 4). First, system engineers develop and maintain the heritage inventory systems. Second, project managers make decisions and supervise data builders including data collectors, data compilers, and engineers. They do this by maintaining communication with external organisations and professionals. $\mathrm{CEDACH}$ plans to develop curricula required for these specialists after the CEDACHeLi 1 launches.

Contents of the CEDACHeLi 1 included an introduction to the concept of disaster-prevention heritage studies, information literacy, and practical tips on information processing. Each unit was 


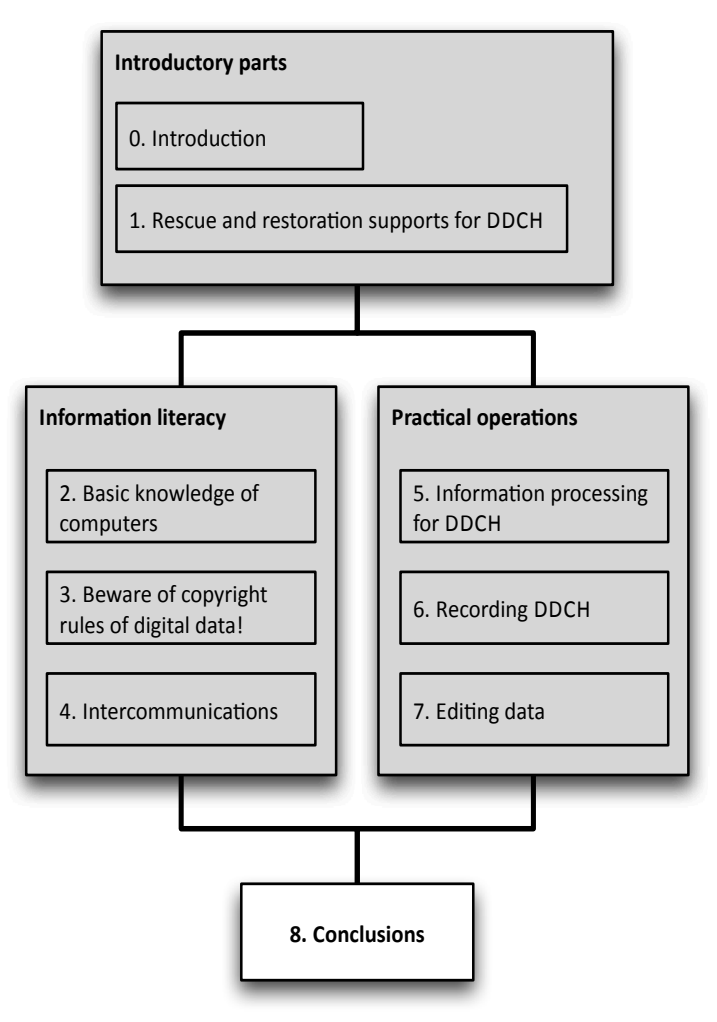

Figure 5: Unit structure of the CEDCHeLi 1; it was designed to provide basic knowledge and practical tips on ICT-based support of rescue operations of disaster-damaged cultural heritage (DDCH)

designed to take 10 minutes to learn to a sufficient level. The units learning objectivities were explicitly presented at the beginning of each unit. This introduction was followed by the main body. Each unit ended with five quizzes to evaluate the learners understanding. The contents were written in Japanese.

The CEDACHeLi 1 comprised approximately 90 units, which were divided into nine chapters. A chapter was divided into two to seven sections, which were then subdivided into several individual units (Figure 5 and Table 1).

Table 1: Curriculum of the CEDACHeLi 1

\section{Introduction}

0.1 . Goals of this e-Learning material

0.2. How to use this e-Learning system

\section{Rescue and restoration supports for DDCH}

1.1. What is DDCH?

1.2. Disaster-related heritage studies

1.3. Rescue operations of DDCH in action

1.4. Necessities for data builders

1.5. Ordinary situations

1.6. Urgent situations

1.6.1. Phase 1: Rescue

1.6.2. Phase 2: Recovery

1.6.3. Phase 3: Restoration

1.7. Role, significance, knowledge and skills

required for data collectors

\section{Basic knowledge of computers}

2.1. What is digital data?

2.1.1. Digital and analog
2.1.2. Encoding and unreadable characters

2.2. How computers work

2.2.1. Roles of operation system

2.2.2. Windows vs. Mac vs. Linux

2.3. Data type

2.3.1. What is character string type?

2.3.2. What is numerical type?

2.3.3. What is date type?

2.4. Directories and placement of files

2.4.1. What is directory?

2.4.2. What is file?

2.4.3. What is extension?

\section{Beware of the copyright rules of digital data!}

3.1. Rights of data source

3.1.1. Public domain

3.1.2. Creative Commons License

3.2. What is a free and open source application?

3.2.1. Concept of free and open source applications

3.2.2. Merits of free and open source applications

3.2.3. Licenses for free and open source applications

\section{Intercomminucations}

4.1. Importance of intercommunications

4.2. E-mail and mailing list

4.3. Groupware (such as Cybozu)

4.4. Video and audio chatting (such as Skype)

4.5. Collecting associated information

\section{Information processing for DDCH}

5.1. $6 \mathrm{~W} 2 \mathrm{H}$ : What is the object for recording?

5.1.1. Damaged cultural property itself

5.1.2. Damaged document and data sheets

5.1.3. Damaged cultural landscape

5.1.4. Testimony

5.2. Sorting collected data

5.3. Types of collected data

5.3.1. Information management based on projects

5.3.2. Text-based information

5.3.3. Summarized information

5.3.4. Multimedia information (pictures, audio and video)

\section{Recoding DDCH}

6.1. Text-based information: diary, data sheets, and field notes

6.1.1. Why do we take notes?

6.1.2. Recording necessary and sufficient information

6.1.3. Taking the protection of personal information into account

6.1.4. How to synchronise other information?

6.2. Visual information

6.2.1. Is audio-visual information the richestt?

6.2.2. How to choose camera: compact or SLR

6.2.3. Configuration of camera settings

6.2.4. Configuration of video camera settings

6.2.5. Composition and subjects

6.2.6. Asking the permission of people to be photographed

6.2.7. Embedding the locational information

6.2.8. Photography notes

6.2.9. Keeping safe

6.3. Audio information

6.3.1. How to use the IC recorder

6.4. Locational information

6.4.1. Logging the location: GPS logger

\section{Editing data}

7.1. What is user-friendly data?

7.2. Management of collected data 
7.2.1. Saving primary data

7.2.2. Processing secondary data

7.2.3. Sharing data with cloud-based services

7.3. Inputting data to forms: maintain a unified format

7.3.1. Reduce the inconsistency of words and formats

7.3.2. Type numerics in English one byte characters

7.3.3. Comment on unformatted items in remarks

7.3.4. Keep descriptions as simple as possible

\section{Conclusions}

8.1. What we have learned in this e-Learning system

8.2. How to pass know-hows and experience to next generations

8.2.1. Review the results of publication

8.2.2. Calling for members working together

8.2.3. Step-up to data managers

\subsubsection{References}

Chapters 0 and 1 introduce the topic (Figure 5 and Table 1). These chapters specify the target learners and present the key concepts of disaster-related heritage studies, methods of conservation, risk management, and education about heritage damaged by past disasters. Learners are expected to understand the reasons why they study disaster-related heritage in Chapter 0 and why they need to acquire knowledge and skills for ICT-based rescue projects of disaster-damaged cultural heritage in Chapter 1.

Chapters 2, 3, and 4 are devoted to developing the information literacy required for ICT-based works. Chapter 2 provides fundamental knowledge of computers, such as operation systems, character encoding, data types, files and directories. Chapter 3 urges learners to pay special attention to licenses and copyrights of digital data and software. Chapter 4 introduces the means of web-based communications, such as e-mail, mailing lists, groupware, and video chatting services. These chapters were written for situations in which several individuals work collaboratively. Therefore, the emphasis was placed on the compliance and intercommunications rather than on how to use computers.

Chapters 5, 6, and 7 provide practical tips on data collection and compilation, which are the main parts of this e-Learning system. Chapter 5 presents types and characteristics of data collected in rescue operations of disaster-damaged cultural heritage based on the experience of CEDACH members. Practical tips in collecting data in the field are then described in Chapter 6, while those for editing data are shown in Chapter 7. Learners are encouraged to study these units in the sequence of the ordinary learning environments, although it is also possible to take units as they like in urgent cases.

An open source learning management system, called Moodle, was employed for online implementation of the CEDACHeLi 1. It was hosted by an application service provider (ASP, see Figure 6). The advantage of ASP is the economic efficiency for e-Learning providers in terms of the low costs of hardware purchases (e.g. servers) and system maintenance. This Internet-based system facilitates both contents development and communications between learners and teachers. Its open source architecture allows for quick creation of working environments in an urgent case at a relatively low cost.

The CEDACHeLi 1 will launch in late 2013. The e-Learning system will continuously be assessed and improved by feedback from learners and professional reviewers. Future work includes promoting the system to potential learners and implementing coursework using the system. We also plan to develop mobile applications making it possible to use the CEDACHeLi in disaster areas on demand, so long as the local mobile telecommunication services are functioning.

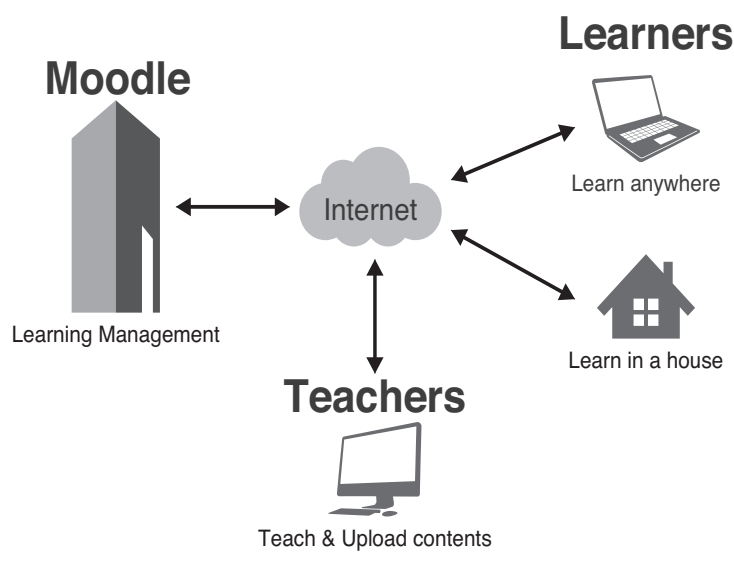

Figure 6: Systematic framework of the CEDACHeLi

\section{CONCLUSIONS}

This paper presented the projects carried out by CEDACH to support the restoration and conservation of the cultural heritage damaged and endangered by the Great East Japan Earthquake in March 2011. Now that two years have passed, it is thought that the importance of support from remote areas is diminishing because of the increasing amount of rescue archaeological work preceding building construction. Restoration projects need more local fieldwork and less remote support. This changing situation makes it increasingly difficult to maintain communication between those working in disaster areas in the Tohoku region in northeastern Japan and the CEDACH office in the Kansai region of western Japan. We believe that this situation is not particularly optimistic. In order to remedy it, we plan to proceed by accomplishing: (1) the publication of the CEDACH GIS and CEDACHeLi; and (2) feedback on the Ishinomaki book catalogue provided to the museum and public with assessments of the workflow and quality of the product.

History of the Japanese archipelago consists of repeated natural disasters (Sangawa, 2009). According to some researchers in geoscience and governmental task forces, it is highly probable that a Great Tokai Earthquake in the Tokai and Kanto regions and a Great Nankai Earthquake in the Kansai region, both along the Pacific coast, will take place within the next years. In the case of the Great East Japan Earthquake, these regions played an important role in providing remote support, but these areas may be the epicenter of disaster areas next time. Facing this possible situation, two questions for researchers in history and archaeology are raised: First, is it possible to accomplish the rescue, preservation, and conservation of damaged heritage with sufficiently scientific quality, in agreement with economicallydamaged local residents in the disaster areas? If so, to what extent can researchers contribute to the sustainable development of this disaster-prone archipelago in a subjective manner, while involving the public and taking into account global issues of disaster risk reduction? We encourage further discussion of this issue with international colleagues, since cases of disaster-related heritage can develop anywhere in the world.

\section{Acknowledgements}

This project is financially supported by the Toyota Foundation (D11-R-0729). The Ishinomaki book catalogue project could not have been implemented without the kind proposal by the Ishinomaki City Board of Education and the Miyagi Shiryounet. The authors would like to thank Dr David Myers for his kind invitation to the Digital Heritage Inventory session of CIPA 2013 and for proofreading the extended abstract. 


\section{REFERENCES}

CEDACH Jimukyoku, 2012. Ishinomaki Bunka Sentā tosho kādo satsuei owaru [Photography of book catalog cards from Ishinomaki Culture Center completed] (in Japanese). CEDACH Newsletter 2, pp. 1-2. http://cedach2.sakura.ne.jp/wpcontent/uploads/2012/07/cedach_news_02.pdf (9 Jun. 2013).

Fujimoto, Y., Kondo, Y., Kaneda, A., Seino, Y., Yamaguchi, H. and Uozu, T., 2011. Organizing diverse and dispersed information on the endangered cultural properties by a voluntary initiative: consortium for the earthquake-damaged cultural heritage (CEDACH). ISPRS SC Newsletter 5(1), pp. 4. http://www.isprssc.org/material/isprs_sc_vol5_nol.pdf (7 Apr. 2013).

Kaner, S., Habu, J. and Matsui, A., 2011. Rescuing archaeology affected by the Japanese earthquake and tsunami. Antiquity. Project Gallery. http://www.antiquity.ac.uk/projgall/kaner329/ (10 Jun. 2013).

Kondo, Y., Kaneda, A., Fujimoto, Y., Seino, Y., Yamaguchi, H. and Uozu, T., 2012. The CEDACH DMT: a volunteer-based data management team for the documentation of the earthquakedamaged cultural heritage in japan. In: M. Zhou, I. Romanowska, Z. Wu, P. Xu and P. Verhagen (eds), Revive the Past: Proceeding of the 39th Conference on Computer Applications and Quantitative Methods in Archaeology, Beijing, 12-16 April 2011, Amsterdam University Press, Amsterdam, pp. 326-333. http://dare.uva.nl/aup/nl/record/412958 (28 Jun. 2013).

Myers, D., Dalgity, A., Avramides, I. and Withrich, D., 2012. Arches: an open source GIS for the inventory and management of immovable cultural heritage. In: M. Ioannides, D. Fritsch, J. Leissner, R. Davies, F. Remondino and R. Caffo (eds), Progress in Cultural Heritage Preservation. 4th International Conference, EuroMed 2012, Limassol, Cyprus, October 29 November 3, 2012. Proceedings, Springer, London, pp. 817-824. doi: 10.1007/978-3-642-34234-9_87.

Okamura, K., Fujisawa, A., Kondo, Y., Fujimoto, Y., Uozu, T., Ogawa, Y. and Mizoguchi, K., 2013. The Great East Japan Earthquake and cultural heritage: towards an archaeology of disaster. Antiquity 87, pp. 258-269.

Sangawa, A., 2009. A study of paleoearthquakes at archeological sites: a new interdisciplinary area between paleoseismology and archaeology. Synthesiology English edition 2(2), pp. 84-94.

Uozu, T. and Seino, Y., 2013. Web sābisu wo riyōshita Ishinomaki Bunka Sentā shozō toshokādo no dētaka sagyō [Digitisation of library catalog cards stored at Ishinomaki Culture Center with web services] (in Japanese). Miyagi Kōkogaku [The Miyagi Archaeology] 15, pp. 9-14. 